\title{
Construction of a 26-feature gene support vector machine classifier for smoking and non-smoking lung adenocarcinoma sample classification
}

\author{
LEI YANG $^{1 *}$, LU SUN $^{2 *}$, WEI WANG $^{1}$, HAO XU $^{1}$, YI LI $^{1}$, JIA-YING ZHAO $^{1}$, \\ DA-ZHONG LIU ${ }^{1}$, FEI WANG ${ }^{1}$ and LIN-YOU ZHANG ${ }^{1}$ \\ ${ }^{1}$ Department of Thoracic Surgery and ${ }^{2}$ The First Cardiac Surgery Department, \\ The Second Affiliated Hospital of Harbin Medical University, Harbin, Heilongjiang 150086, P.R. China
}

Received May 2, 2017; Accepted October 4, 2017

DOI: $10.3892 / \mathrm{mmr} .2017 .8220$

\begin{abstract}
The present study aimed to identify the feature genes associated with smoking in lung adenocarcinoma (LAC) samples and explore the underlying mechanism. Three gene expression datasets of LAC samples were downloaded from the Gene Expression Omnibus database through pre-set criteria and the expression data were processed using meta-analysis. Differentially expressed genes (DEGs) between LAC samples of smokers and non-smokers were identified using limma package in $\mathrm{R}$. The classification accuracy of selected DEGs were visualized using hierarchical clustering analysis in $\mathrm{R}$ language. A protein-protein interaction (PPI) network was constructed using gene interaction data from the Human Protein Reference Database for the DEGs. Betweenness centrality was calculated for each node in the network and genes with the greatest BC values were utilized for the construction of the support vector machine (SVM) classifier. The dataset GSE43458 was used as the training dataset for the construction and the other datasets (GSE12667 and GSE10072) were used as the validation datasets. The classification accuracy of the classifier was tested using sensitivity, specificity, positive predictive value, negative predictive value and area under curve parameters with the pROC package in R language. The feature genes in the SVM classifier were subjected to pathway enrichment analysis using Fisher's exact test. A total of 347 genes were identified to be differentially expressed between samples of smokers and non-smokers. The PPI network of DEGs were
\end{abstract}

Correspondence to: Dr Lin-You Zhang, Department of Thoracic Surgery, The Second Affiliated Hospital of Harbin Medical University, 246 Xuefu Road, Harbin, Heilongjiang 150086, P.R. China E-mail: lyzhang6696@126.com

\section{${ }^{*}$ Contributed equally}

Key words: lung adenocarcinoma, smoking, feature genes, protein-protein interaction network, support vector machine classifier comprised of 202 nodes and 300 edges. An SVM classifier comprised of 26 feature genes was constructed to distinguish between different LAC samples, with prediction accuracies for the GSE43458, GSE12667 and GSE10072 datasets of 100, 100 and $94.83 \%$, respectively. Furthermore, the 26 feature genes that were significantly enriched in 9 overrepresented biological pathways, including extracellular matrix-receptor interaction, proteoglycans in cancer, cell adhesion molecules, p53 signaling pathway, microRNAs in cancer and apoptosis, were identified to be smoking-related genes in LAC. In conclusion, an SVM classifier with a high prediction accuracy for smoking and non-smoking samples was obtained. The genes in the classifier may likely be the potential feature genes associated with the development of patients with LAC who smoke.

\section{Introduction}

Lung cancer is the most common cause of cancer-associated fatality in men and the second most common in women (1). The 5-year survival rate following diagnosis of lung cancer is $15.6 \%$, making it one of the worst prognostic malignant tumors (2). The survival rate is lower compared with breast, colon and prostate cancer (2). Cigarette smoking is responsible for $\sim 90 \%$ of lung cancer incidences and leads to decreased survival rates (3).

The major histological types of lung cancer include adenocarcinoma, squamous cell carcinoma, large cell carcinoma and small cell carcinoma. The incidence of lung adenocarcinoma (LAC) increased gradually and this lung cancer has been the most frequently occurring histological type in most parts of the world in recent years (4). Adenocarcinoma account for $\sim 40 \%$ of all lung cancer cases (5). Smoking is a major cause of lung adenocarcinoma (6). However, the causes of the increase in adenocarcinomas are not clear.

Sequencing data from large-scale databases, such as The Cancer Genome Atlas, have aided in identification of novel factors and potentially targetable alterations in lung adenocarcinomas (7). A number of smoking-associated genes have been revealed in LAC, including the cyclin D1 A870 G gene, and polymorphisms of this gene have been indicated to modulate smoking-induced lung cancer risk (8). 
Estrogen receptor $\alpha$ promotes smoking carcinogen-induced lung carcinogenesis via cytochrome P450 1B1 (9). The interactions between smoking, polymorphisms of human 8-oxoguanine DNA glycosylase and p53 are associated with the development of lung cancer (10). Interactions between smoking, fragile histidine triad gene alterations (11) and excision repair cross-complementation group 1 polymorphisms (12) have also been reported in lung cancer. However, the recognized genetic changes in patients with LAC who are smokers remain to be elucidated and further studies are necessary to determine the underlying molecular mechanism of smoking-induced LAC.

A recent study has aimed to identify smoking-associated genes via the differential analysis of RNA sequencing data (13). The study analyzed two datasets with only two samples and identified 1,603 differentially expressed genes (DEGs). The authors also identified that the possible alternative splicing of gene FCGBP may have an impact on lung cancer. However, the small sample size could lead to low reliability of the results.

In the present study, three gene expression datasets of smokers and non-smokers with LAC (>50 samples/group) were obtained and DEGs were identified using meta-analysis. A protein-protein interaction (PPI) network of the DEGs was constructed with the betweenness centrality (BC) analysis for the selection of feature genes. Using the feature genes, a support vector machine (SVM) classifier, which is able to distinguish between samples from smokers and non-smokers with a high classification accuracy, was constructed. The feature genes in the SVM classifier were considered as the smoking-related genes in LAC and enrichment analysis was conducted to identify significant pathways.

\section{Materials and methods}

Gene expression data. To collect gene expression data from patients with LAC who smoke or do not smoke, the Gene Expression Omnibus (GEO; www.ncbi.nlm.nih.gov/geo/) database was used and the key words 'lung adenocarcinoma', 'Homo sapiens' and 'smoke' were searched. The following inclusion criteria were used to extract the corresponding datasets: i) They were gene expression data; ii) they were from LAC samples; iii) information concerning smoking was described; and iv) $\geq 50$ samples were included in each dataset. A total of three datasets were collected from the GEO database, including GSE43458 (14), GSE10072 (15) and GSE12667 (16) (Table I).

Raw data in these three datasets were analyzed with the affy package in R 3.2.1 (http://bioconductor.org/packages/ release/bioc/html/affy.html) (17). Probes were subsequently mapped into genes. Probes corresponding to one gene were averaged as the final expression value of the gene. Normalization was performed with package limma (18) of R to conduct the analysis of the datasets.

Screening of DEGs. Meta-analysis was used to enforce the analytical reliability for gene expression data by combining data from different datasets. DEGs associated with smoking in the three gene expression datasets were screened via meta-analysis using the MetaDE.ES package of R (19). The method tested the heterogeneity of gene expression value from three datasets with three statistic parameters: $\mathrm{Tau}^{2}$, Q-value and Qpval. Subsequently, differential expression of genes between smoking and non-smoking samples was assessed by determining the P-value and false discovery rate (FDR). To determine the DEGs associated with smoking, $\operatorname{tau}^{2}=0$, Qpval $>0.05$ and FDR $<0.05$ were set as the cut-off points. Bidirectional clustering analysis using the pheatmap package in $\mathrm{R}$ language (https://cran.r-project. org/web/packages/pheatmap/index.html), which was based on the euclidean distance calculations for gene expression values, was also conducted to examine whether the selected DEGs were able to distinguish different samples, as described previously (20).

Construction of PPI network. To investigate the interactions of DEGs, the DEGs were mapped to the PPI database using the Human Protein Reference Database (21). The interactions of DEGs obtained were constructed into a PPI network with the proteins that were connected with at least three DEGs. The network was visualized with Cytoscape (22).

Calculation of $B C$. Feature genes that function as hub nodes in the PPI network were screened using a BC algorithm (23). $\mathrm{BC}$ represented the degree of node in the network and was calculated as follows:

$$
\mathrm{C}_{\mathrm{B}}(v)=\sum_{t \neq v \neq u \in V} \frac{\sigma_{s t}(v)}{\sigma_{s t}}
$$

Where ost is the total number of shortest paths from node $s$ to node $t ; \sigma s t(v)$ is the number of shortest paths from $s$ to $t$ going through $v$; $\mathrm{BC}$ scores were between 0 and 1 , and a higher $\mathrm{BC}$ score indicated a higher degree of the node.

Training and validation of SVM classifier. SVM classifier comprises of feature genes that distinguishes between different samples $(24,25)$. To construct the SVM classifier, one of the downloaded datasets, GSE43458 (containing 40 non-smokers and 40 smokers) was selected as the training dataset basing on the top 10, 20, 30, 40 and 50 feature genes ranked by BC scores. The feature genes in the SVM classifier that could exactly distinguish between different samples in GSE42458 were subjected to two-way clustering analysis using pheatmap package in $\mathrm{R} 3.1 .4$ (https://cran.r-project. org/web/packages/pheatmap/index.html). Sample similarity matrices were also obtained by computing the Pearson's correlation coefficients of these genes using Cor package in R 3.1.4 (https://stat.ethz.ch/R-manual/R-devel/library/stats/html/cor. html) and top 50 genes were selected for further analysis. The clustering and similarity matrices were visualized using heatmaps in pheatmap package in R 3.1.4 (https://cran.r-project. org/web/packages/pheatmap/index.html).

The SVM classifier was validated with two independent datasets, GSE10072 and GSE12667. Sensitivity (Se), specificity (Sp), positive predictive value (PPV), negative predictive value (NPV) and area under curve (AUC) were calculated using the pROC package in R language (https://cran.r-project. org/web/packages/pROC/index.html) to examine the classification accuracy of the SVM classifier as described previously $(26,27)$. 
Table I. Data of the three collected gene expression datasets.

\begin{tabular}{llccc}
\hline Accession number & \multicolumn{1}{c}{ Platform } & Total samples (n) & Non-smokers (n) & Smokers (n) \\
\hline GSE43458 & HuGene-1_0-st-v1 & 110 & 40 & 40 \\
GSE10072 & HG-U133A & 107 & 16 & 42 \\
GSE12667 & HG-U133_Plus_2 & 75 & 8 & 43 \\
\hline
\end{tabular}

Table II. Top 10 candidate feature genes by FDR.

\begin{tabular}{|c|c|c|c|c|c|c|}
\hline ID & P-value & FDR & $\operatorname{tau}^{2}$ & Qpval & Qval & Expression \\
\hline ABCB11 & $1.52 \times 10^{-05}$ & $5.59 \times 10^{-04}$ & 0 & $9.27 \times 10^{-01}$ & $8.28 \times 10^{-03}$ & $\mathrm{Up}$ \\
\hline ABCB6 & $2.27 \times 10^{-03}$ & $2.23 \times 10^{-02}$ & 0 & $9.63 \times 10^{-01}$ & $2.18 \times 10^{-03}$ & $\mathrm{Up}$ \\
\hline $\mathrm{ABCC} 2$ & $2.11 \times 10^{-03}$ & $2.11 \times 10^{-02}$ & 0 & $9.51 \times 10^{-01}$ & $3.83 \times 10^{-03}$ & $\mathrm{Up}$ \\
\hline ABCG5 & $4.01 \times 10^{-06}$ & $2.10 \times 10^{-04}$ & 0 & $9.00 \times 10^{-01}$ & $1.58 \times 10^{-02}$ & Up \\
\hline $\mathrm{ACD}$ & $4.81 \times 10^{-06}$ & $2.38 \times 10^{-04}$ & 0 & $9.40 \times 10^{-01}$ & $5.71 \times 10^{-03}$ & Up \\
\hline ADAMTS5 & $1.88 \times 10^{-04}$ & $3.79 \times 10^{-03}$ & 0 & $9.10 \times 10^{-01}$ & $1.26 \times 10^{-02}$ & $\mathrm{Up}$ \\
\hline AGT & $2.89 \times 10^{-03}$ & $2.64 \times 10^{-02}$ & 0 & $8.47 \times 10^{-01}$ & $3.71 \times 10^{-02}$ & $\mathrm{Up}$ \\
\hline AIM1L & $1.00 \times 10^{-20}$ & $7.47 \times 10^{-19}$ & 0 & $8.26 \times 10^{-01}$ & $4.84 \times 10^{-02}$ & $\mathrm{Up}$ \\
\hline AKAP6 & $1.15 \times 10^{-04}$ & $2.69 \times 10^{-03}$ & 0 & $9.82 \times 10^{-01}$ & $5.08 \times 10^{-04}$ & Down \\
\hline ALPL & $5.01 \times 10^{-03}$ & $3.88 \times 10^{-02}$ & 0 & $9.06 \times 10^{-01}$ & $1.40 \times 10^{-02}$ & Down \\
\hline
\end{tabular}

FDR, false discovery rate; UP, upregulation in smokers; DOWN, downregulation in smokers.

Pathway enrichment analysis. Feature gene-related Kyoto Encyclopedia of Genes and Genomes pathways (http://www. genome.jp/kegg/) were revealed using Fisher's exact test as follows:

$$
\mathrm{p}=1-\sum_{i=0}^{x-1} \frac{\left(\begin{array}{l}
\mathrm{M} \\
\mathrm{i}
\end{array}\right)\left(\begin{array}{l}
\mathrm{N}-\mathrm{M} \\
\mathrm{K}-\mathrm{i}
\end{array}\right)}{\left(\begin{array}{l}
\mathrm{N} \\
\mathrm{K}
\end{array}\right)}
$$

Where $N$ represented the total number of genes; $M$ represented the number of genes in the pathway; and $K$ indicated the number of feature genes.

\section{Results}

DEGs. A total of 12,476 genes were in the three gene expression datasets, and according to the set criteria, 347 DEGs between smoking and non-smoking LAC samples were identified. The top 10 DEGs ranked by FDR are listed in Table II. As indicated in Fig. 1, the 347 DEGs distinguished the samples of smokers from the non-smokers.

PPI network. A PPI network containing 202 nodes (genes) and 300 edges (connection between nodes) was obtained (Fig. 2). The proteins that were connected with $\geq 3$ DEGs were also included in the PPI network. Degree distribution of genes in the network is indicated in Fig. 3. Similar to biological networks, the PPI network was scale-free, with the majority of genes (80 genes) exhibiting small degrees (Log transformed degree $<1$ ) and few genes (only 5) exhibiting larger degrees (Log transformed degree between 3 and 4). The genes with high degrees were hub genes, indicating their roles in the development of smoking-associated LAC.
Feature genes. BC was calculated for each node in the PPI network. The top 10 genes by $\mathrm{BC}$ value were considered as the feature genes, including high mobility group box 1 (HMGB1); dynein light chain LC8-type 1; tubulin $\alpha$ 4a; 14-3-3 protein $\gamma$; tyrosine 3 -monooxygenase; spectrin $\beta$, non-erythrocytic 1 ; ubiquilin 4; DNA methyltransferase 1 (DNMT1); enhancer of zeste 2 polycomb repressive complex 2 subunit (EZH2) and glucocorticoid modulatory element binding protein 1 (Table III).

SVM classifier. Feature genes with the greatest BC values were used to construct the SVM classifier basing on dataset GSE43458. There were 8, 11, 14, 16, 18, 20, 22 and 26 feature genes in the top 10, 15, 20, 25, 30, 35, 40 and 50 genes, respectively. The training process is indicated in Fig. 4. The accuracy of the classifier reached $100 \%$ when the 26 feature genes in the top 50 were included. Therefore, the classifier comprised by these 26 feature genes were chosen as the final SVM classifier. These feature genes included $\mathrm{Cbl}$ proto-oncogene $\mathrm{B}$ (CBLB), DNMT1, EZH2, HMGB1, integrin $\alpha-5$ (ITGA5), MDK, protein kinase $\mathrm{C} \iota(\mathrm{PRKCI})$ and sprouty receptor tyrosine kinase signaling antagonist 2 (SPRY2).

Hierarchical clustering was performed for samples from the training dataset using the 26 feature genes (Fig. 5). The classifier separated samples of smokers from samples of non-smokers in dataset GSE43458 (Fig. 6A).

The SVM classifier was validated using dataset GSE12667 and GSE10072. The classification accuracy in GSE12667 was $100 \%$ (Fig. 6B). In GSE10072, the classifier identified 42 smokers $(42 / 42,100 \%)$ and 13 non-smokers (13/16, 81.25\%), and total accuracy was $94.83 \%$ (55/58) (Fig. 6C; Table IV). The classifier demonstrated high accuracy of 100, 100 and $94.83 \%$ 


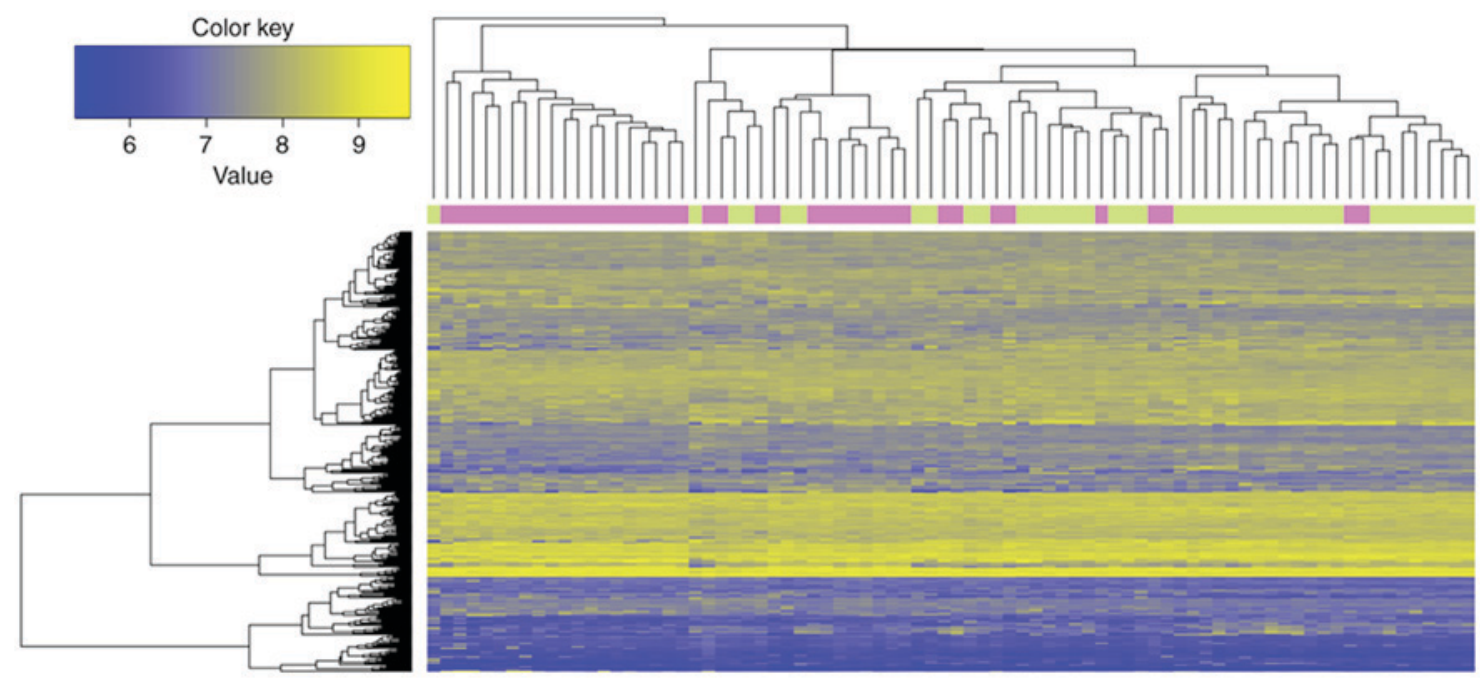

Figure 1. Hierarchical clustering results of lung adenocarcinoma samples from smokers and non-smokers according to the 347 differentially expressed genes. $\mathrm{X}$-axis represents samples, in which samples of smokers are in purple whereas samples of non-smokers are in green; $y$-axis represents differentially expressed genes.

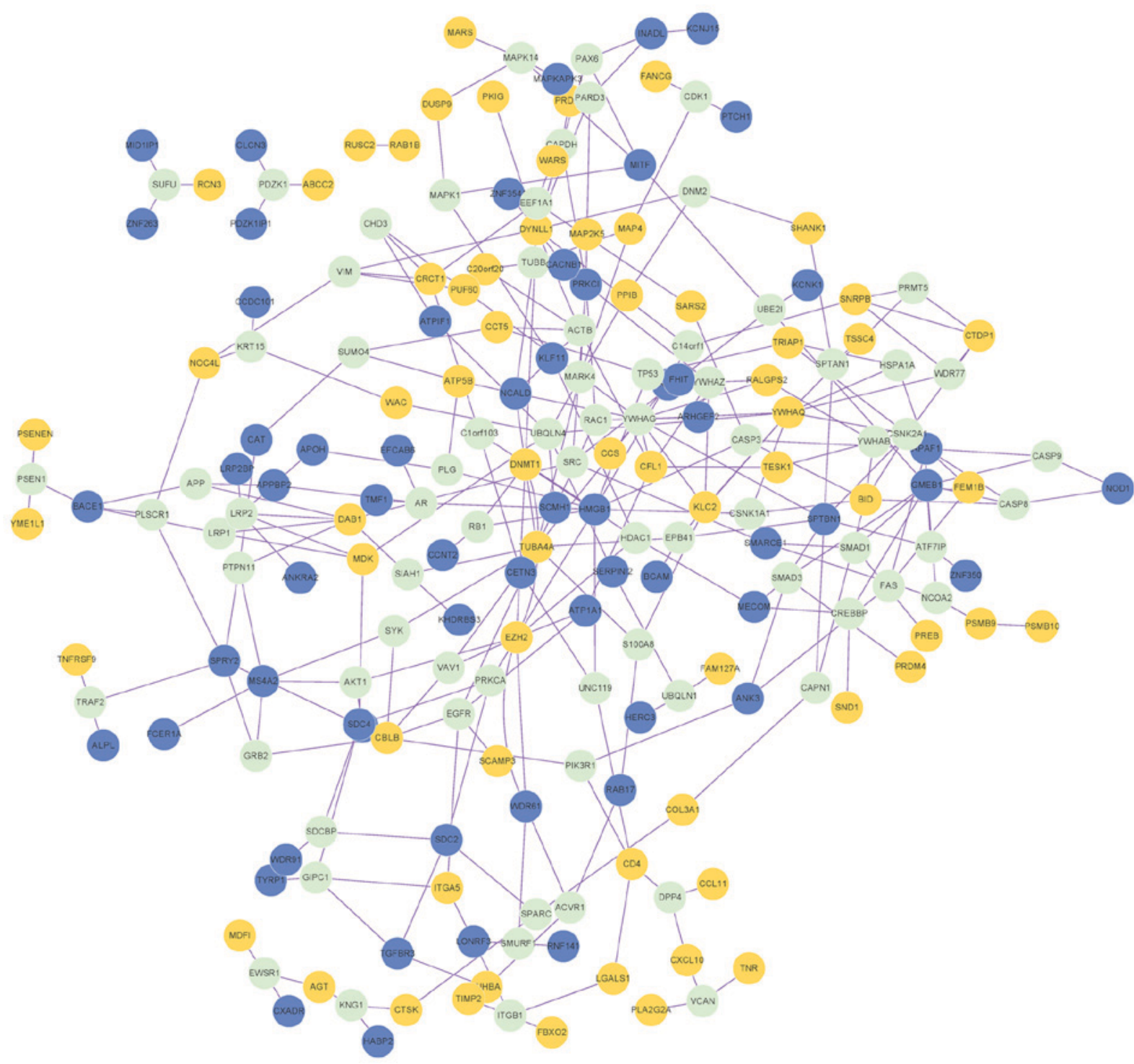

Figure 2. PPI network of differentially expressed genes identified between lung adenocarcinoma samples of smokers and non-smokers. Differentially expressed genes were differentially expressed in samples of smokers compared with samples of non-smokers. Upregulated genes are marked in orange, downregulated genes are marked in blue. Non-differentially expressed genes that interacted with $\geq 3$ differentially expressed genes were also included in the PPI network. Non-differentially expressed genes are marked in green. PPI, protein-protein interaction. 
Table III. Top 10 genes ranked using BC.

\begin{tabular}{|c|c|c|c|c|c|c|c|}
\hline Gene & $\mathrm{BC}$ & Expression & Degree & P-value & FDR & Qpval & Qval \\
\hline HMGB1 & $1.98 \times 10^{-01}$ & Down & 11 & $1.63 \times 10^{-03}$ & $1.76 \times 10^{-02}$ & $8.95 \times 10^{-01}$ & $1.74 \times 10^{-02}$ \\
\hline DYNLL1 & $1.77 \times 10^{-01}$ & Up & 15 & $1.00 \times 10^{-20}$ & $7.47 \times 10^{-19}$ & $8.92 \times 10^{-01}$ & $1.83 \times 10^{-02}$ \\
\hline TUBA4A & $1.37 \times 10^{-01}$ & $\mathrm{Up}$ & 10 & $2.08 \times 10^{-05}$ & $7.32 \times 10^{-04}$ & $8.50 \times 10^{-01}$ & $3.56 \times 10^{-02}$ \\
\hline YWHAG & $1.20 \times 10^{-01}$ & - & 11 & $9.86 \times 10^{-01}$ & $9.95 \times 10^{-01}$ & $5.38 \times 10^{-06}$ & $2.07 \times 10$ \\
\hline YWHAQ & $1.07 \times 10^{-01}$ & Up & 10 & $2.40 \times 10^{-04}$ & $4.55 \times 10^{-03}$ & $8.46 \times 10^{-01}$ & $3.77 \times 10^{-02}$ \\
\hline SPTBN1 & $1.04 \times 10^{-01}$ & Down & 7 & $9.39 \times 10^{-04}$ & $1.23 \times 10^{-02}$ & $8.53 \times 10^{-01}$ & $3.43 \times 10^{-02}$ \\
\hline UBQLN4 & $1.00 \times 10^{-01}$ & - & 7 & $9.31 \times 10^{-01}$ & $9.67 \times 10^{-01}$ & $5.95 \times 10^{-02}$ & $3.55 \times 10^{0}$ \\
\hline DNMT1 & $9.98 \times 10^{-02}$ & Up & 7 & $1.20 \times 10^{-04}$ & $2.77 \times 10^{-03}$ & $8.61 \times 10^{-01}$ & $3.05 \times 10^{-02}$ \\
\hline $\mathrm{EZH} 2$ & $8.51 \times 10^{-02}$ & $\mathrm{Up}$ & 8 & $2.65 \times 10^{-03}$ & $2.48 \times 10^{-02}$ & $8.44 \times 10^{-01}$ & $3.89 \times 10^{-02}$ \\
\hline GMEB1 & $8.45 \times 10^{-02}$ & Down & 8 & $2.31 \times 10^{-04}$ & $4.40 \times 10^{-03}$ & $9.32 \times 10^{-01}$ & $7.20 \times 10^{-03}$ \\
\hline
\end{tabular}

BC, betweenness centrality; UP, upregulation in smokers; DOWN, downregulation in smokers; -, no significant difference in expression; FDR, false discovery rate.

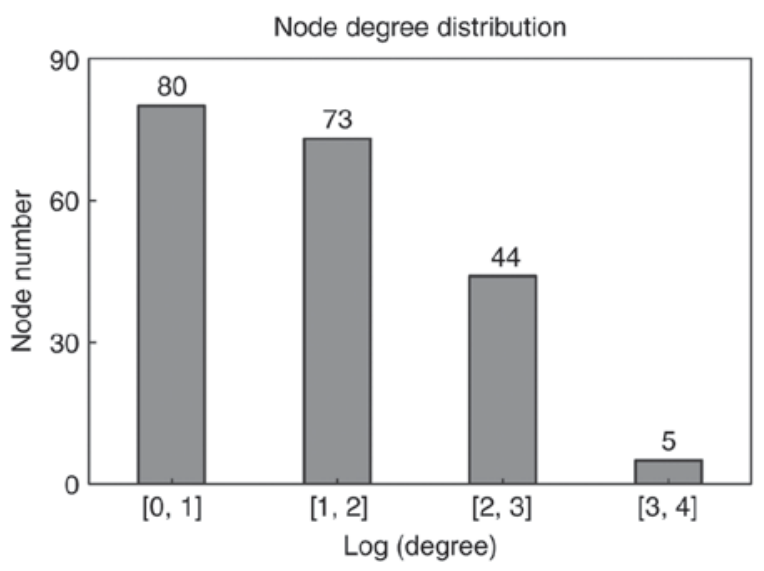

Figure 3. Degree distribution of nodes (genes) in the protein-protein interaction network of differentially expressed genes. $x$-axis indicates the Log transformed degree and $y$-axis indicates the number of nodes.

in GSE43458, GSE12667 and GSE10072, respectively. Se, Sp, PPV, NPV and AUC results (Table IV) and receiver operating characteristic curves were generated (Fig. 7).

Overrepresented biological pathways. The 26 feature genes were indicated to be significantly enriched in nine biological pathways (Table V): Extracellular matrix (ECM)-receptor interaction, proteoglycans in cancer, cell adhesion molecules, pathogenic Escherichia coli infection, p53 signaling pathway, microRNAs in cancer, bacterial invasion of epithelial cells, apoptosis and hematopoietic cell lineage.

\section{Discussion}

In the present study, three gene expression datasets were obtained and a total of 347 DEGs were identified in samples from smokers with LAC compared with non-smokers with LAC using meta-analysis. A PPI network including 202 nodes and 300 edges was constructed, from which 26 feature genes were identified. The SVM classifier of these 26 genes separated smokers from non-smokers with an accuracy $>94 \%$ in all

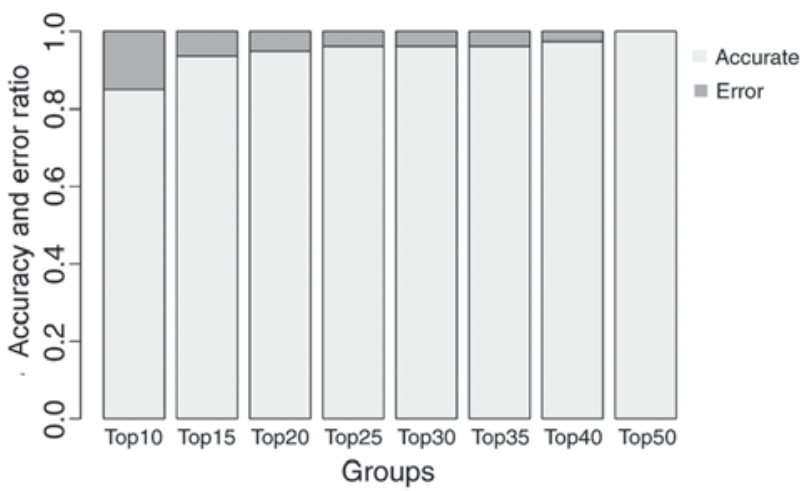

Figure 4. Predictive accuracy and error ratios of support vector machine classifier with different numbers of feature genes. Accuracy is indicated in light gray whereas error rate is indicated in dark gray.

the three datasets. Pathway enrichment analysis demonstrated that these feature genes were primarily associated with cancer development- and metastasis-associated pathways, including ECM-receptor interaction, proteoglycans in cancer, cell adhesion molecules, p53 signaling pathway, microRNAs in cancer and apoptosis.

Due to the generalization ability, SVM has been widely used for analysis, including data classification and function approximation (28-30). SVM classifier has been demonstrated to distinguish whether one cancer sample type possessed distinctive signatures of gene expressions compared with other sample types (31). In the present study, an SVM classifier with 26 feature genes successfully distinguished LAC samples of smokers and non-smokers using bioinformatics analysis. Yousef et al (32) previously conducted a similar study for the identification of biomarkers, by integrating interaction networks and an SVM classifier, and subsequently obtained $>90 \%$ accuracy in classification of selected microarray datasets. Furthermore, a previous study also demonstrated that the discriminant analysis based on an SVM classifier achieved satisfactory results in the classification of lung cancer samples (33).

Specific genes within the 26 feature genes have been implicated in lung cancer or LAC. CBLB is a regulator 

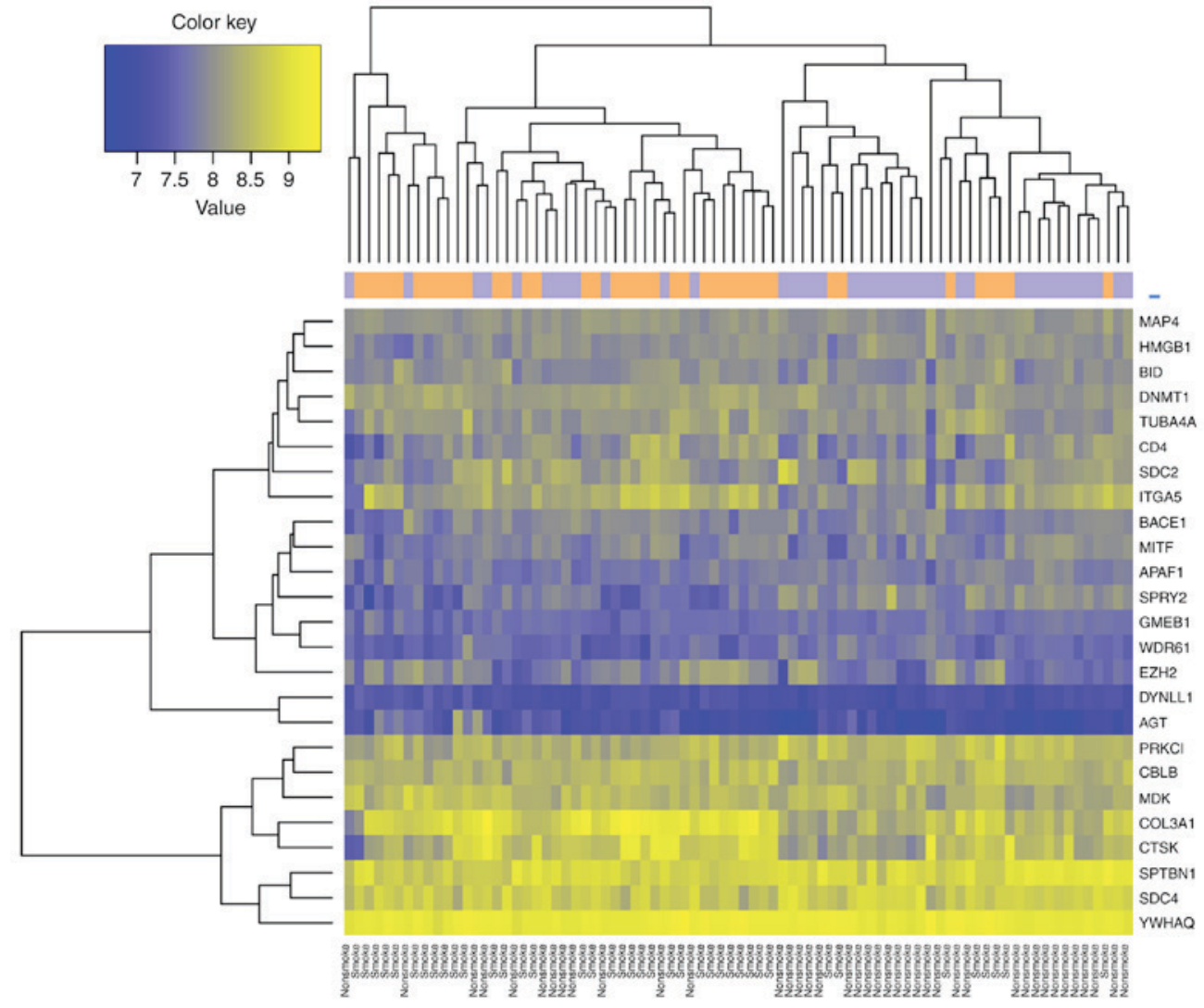

Figure 5. Hierarchical clustering result from samples from smokers and non-smokers with lung adenocarcinoma using the 26 feature genes. $\mathrm{x}$-axis represents samples, in which smokers were marked in orange and non-smokers were marked in purple; y-axis represents the 26 feature genes.
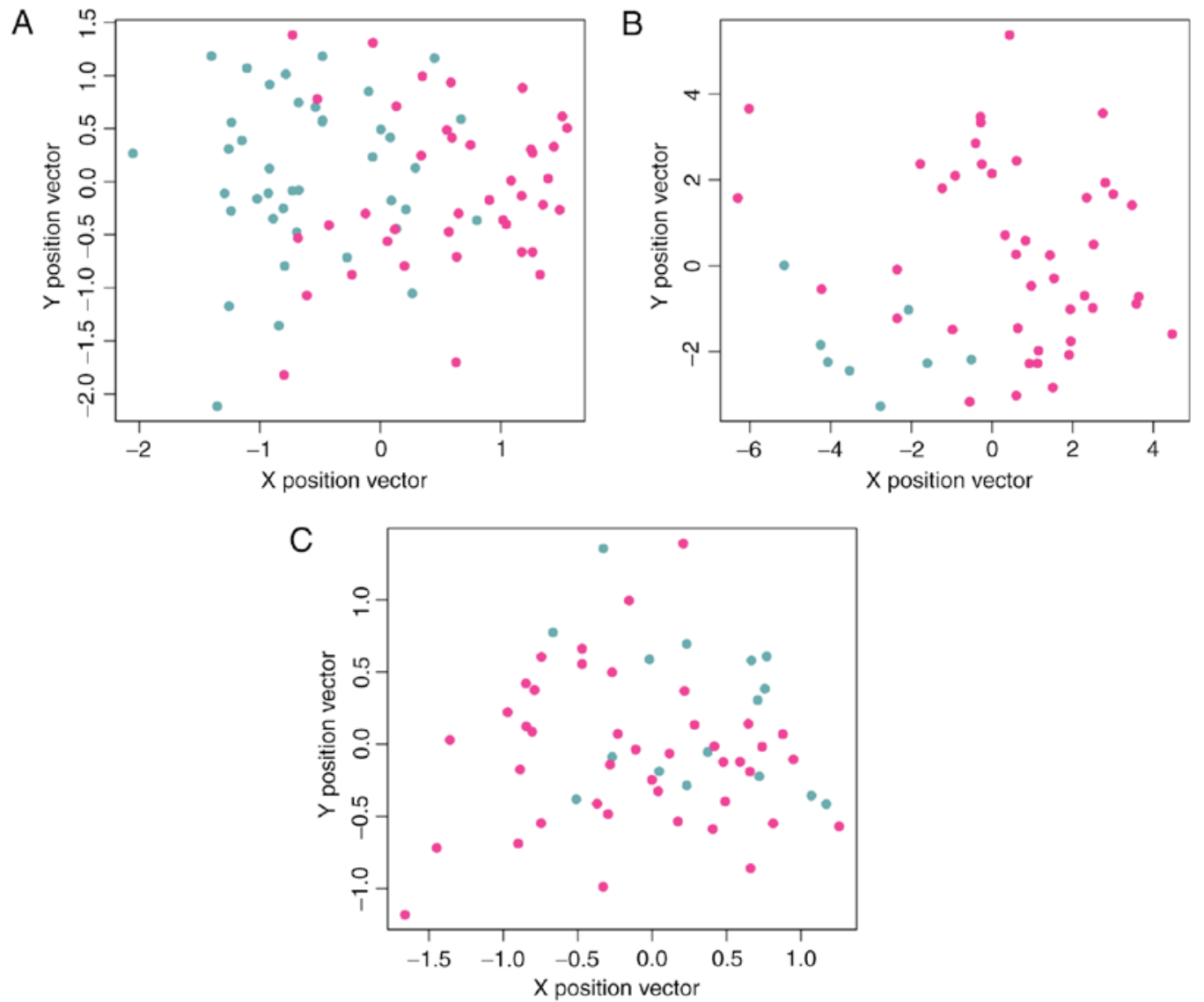

Figure 6. Scatter plots of the support vector machine classifier on three microarray datasets. (A) GSE43458, (B) GSE12667, and (C) GSE10072 microarray datasets were indicated. Smokers are marked in red and non-smokers are marked in green. 
Table IV. Prediction results of the support vector machine classifier in the three datasets.

\begin{tabular}{|c|c|c|c|c|c|c|c|}
\hline Dataset & Samples (n) & Accuracy $(\%)$ & $\mathrm{Se}$ & $\mathrm{Sp}$ & PPV & NPV & AUC \\
\hline GSE43458 & 80 & 100 & 1 & 1 & 1 & 1 & 1 \\
\hline GSE12667 & 51 & 100 & 1 & 1 & 1 & 1 & 1 \\
\hline GSE10072 & 58 & 94.83 & 1 & 0.813 & 0.933 & 1 & 0.994 \\
\hline
\end{tabular}

Se, sensitivity; Sp, specificity; PPV, positive predictive value; NPV, negative predictive value; AUC, area under curve.

Table V. A total of 9 biological pathways significantly overrepresented by the 26 feature genes.

\begin{tabular}{llll}
\hline ID & \multicolumn{1}{c}{ Term } & P-value & \multicolumn{1}{c}{ Genes } \\
\hline hsa04512 & Extracellular matrix-receptor interaction & $5.68 \times 10^{-03}$ & SDC4, ITGA5, COL3A1 \\
hsa05205 & Proteoglycans in cancer & $9.82 \times 10^{-03}$ & SDC4, CBLB, ITGA5, SDC2 \\
hsa04514 & Cell adhesion molecules & $2.17 \times 10^{-02}$ & SDC4, CD4, SDC2 \\
hsa05130 & Pathogenic Escherichia coli infection & $2.19 \times 10^{-02}$ & YWHAQ, TUBA4A \\
hsa04115 & p53 signaling pathway & $3.21 \times 10^{-02}$ & APAF1, BID \\
hsa05206 & MicroRNAs in cancer & $3.29 \times 10^{-02}$ & EZH2, SPRY2, ITGA5, DNMT1 \\
hsa05100 & Bacterial invasion of epithelial cells & $3.91 \times 10^{-02}$ & CBLB, ITGA5 \\
hsa04210 & Apoptosis & $4.86 \times 10^{-02}$ & APAF1, BID \\
hsa04640 & Hematopoietic cell lineage & $4.96 \times 10^{-02}$ & CD4, ITGA5 \\
\hline
\end{tabular}
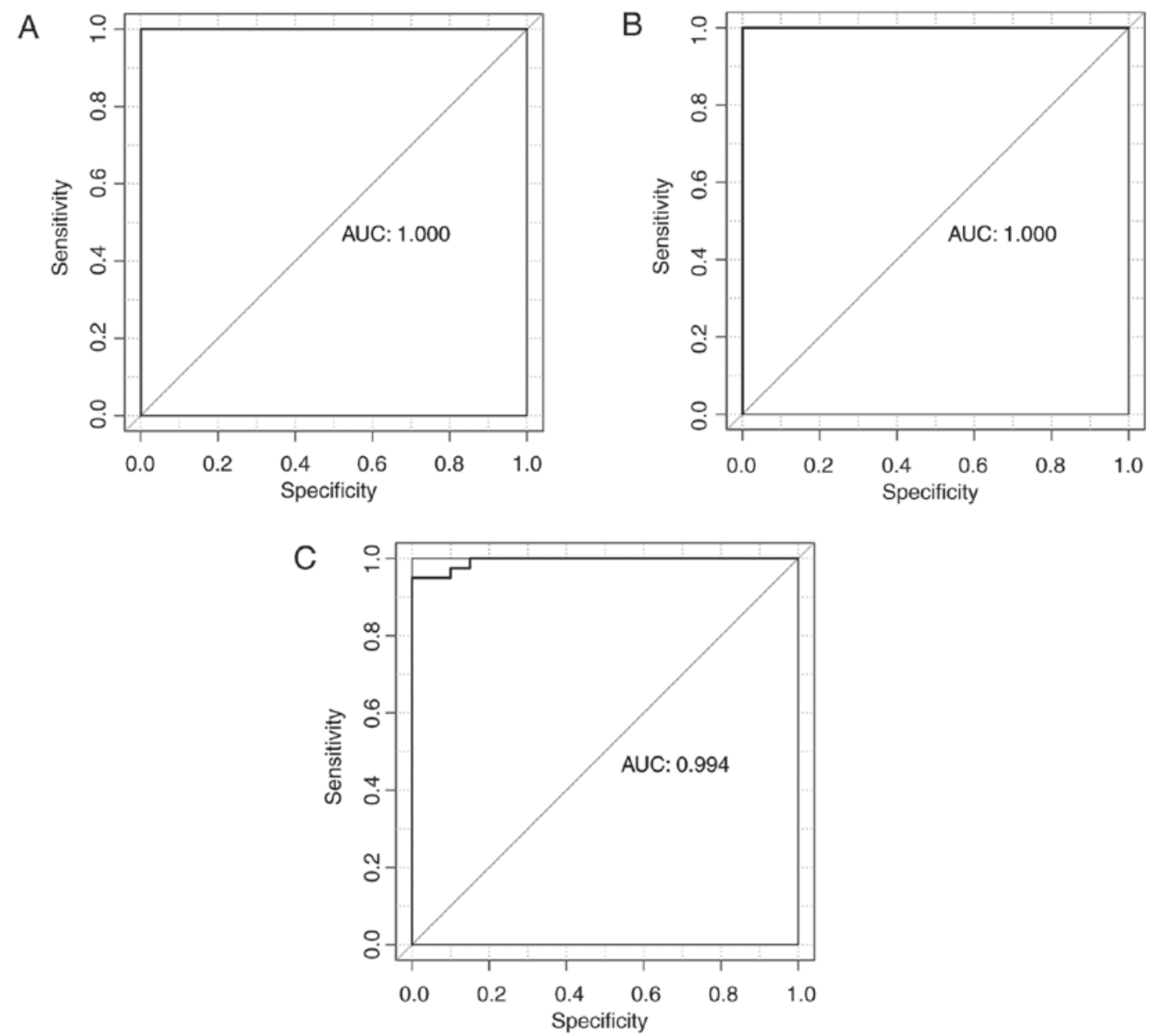

Figure 7. Receiver operating characteristic curves of support vector machine classifier for the three microarray datasets. (A) GSE43458, (B) GSE12667 and (C) GSE10072 microarray datasets were indicated. AUC, area under curve. 
of T-cell response (34). It has been reported that the single nucleotide polymorphisms of CBLB may predict the definitive radiotherapy outcomes for non-small cell lung cancer (NSCLC) (34). CBLB is associated with icotinib-induced apoptosis and G1 phase arrest of epidermal growth factor receptor mutation-positive NSCLC (35).

DNMT1 is responsible for maintaining methylation patterns following DNA replication and has an important role in the development of various types of cancer (36). DNA methylation alterations are recognized as key epigenetic changes in cancer, influencing the chromosomal instability through global hypomethylation and aberrant gene expression via the alterations in methylation levels (37). The tobacco-specific carcinogen nicotine-derived nitrosamine ketone induces the accumulation of DNMT1 in patients with lung cancer (38). Furthermore, DNMT1 inhibits the expression of, the tumor suppressor Wnt7a in NSCLC (39).

EZH2 is a member of the polycomb-group family, which is associated with maintaining the transcriptional repressive state of genes over successive cell generations (40). Yoon et al (41) previously suggested a correlation between the genotype variants in EZH2 and reduced lung cancer risk. Additionally, Zhang et al (42) determined that miR-138 inhibited tumor growth through the repression of EZH2 in NSCLC. Notably, a recent study indicated that EZH2 silencing with RNA interference induced $\mathrm{G} 2 / \mathrm{M}$ arrest in human lung cancer cells in vitro (43), and Wang et al (44) recently demonstrated that EZH2 overexpression was associated with a poor prognosis for patients with LAC. In the present study, it was indicated that EZH2 was upregulated in the samples of smokers and thus the present findings suggest that EZH2 upregulation may result from smoking.

HMGB1 has a role in tumor cell migration (45). Shen et al (46) indicated that the expression of HMGB1 correlates with the progression of NSCLC. ITGA5 is considered as a prognostic indicator in NSCLC (47).

MDK promotes cell growth, migration and angiogenesis, in particular during tumorigenesis (48). A previous study indicated that MDK protein overexpression is correlated with the malignant status and prognosis of NSCLC (49). Furthermore, MDK has been targeted as a therapeutic biomarker for lung cancer (50).

PRKCI is required for lung tumorigenesis as genetic loss of PRKCI inhibits Kras-initiated hyperplasia and subsequent lung tumor formation in vivo (51). SPRY2 inhibits cell migration and proliferation in NSCLC (52). In addition, a previous study has indicated that downregulation of SPRY2 in NSCLC contributes to tumor malignancy (53).

Smoking can cause LAC and the incidence of this disease increased in recent years (4). However, the reason for this increase and the mechanism underlying smoking-associated development of LAC remain to be elucidated. The present study identified genes implicated in smoking-associated LAC, including CBLB, DNMT1, EZH2, HMGB1, ITGA5, MDK, PRKCI and SPRY2. Most of these genes have been reported in association with malignancy and certain were associated with lung cancer. The identification of these characteristic genes may aid in elucidating the mechanism underlying smoking associated-lung adenocarcinoma. Although further experiments such as validation the gene and protein expression level in the smoking and non-smoking LAC samples were not performed limited by the LAC samples available, these results may provide information to other researchers in the field.

In conclusion, a number of key genes have been revealed in smokers with LAC and some of these have been implicated in lung cancer. However, the associations between the 26 feature genes, smoking and LAC remain to be fully elucidated with further studies.

\section{Acknowledgements}

The present study was supported by The Natural Science Foundation of Heilongjiang Province (The Science Foundation for Returned Overseas Students in Heilongjiang Province; grant nos. LC2016036 and LC2015040) and Research Foundation of Harbin Medical University Affiliated Second Hospital (grant nos. KYBS2015-10 and KYBS2015-01).

\section{References}

1. Centers for Disease Control and Prevention (CDC): State-specific trends in lung cancer incidence and smoking-United States, 1999-2008. MMWR Morb Mortal Wkly Rep 60: 1243-1247, 2011.

2. Nanavaty P, Alvarez MS and Alberts WM: Lung cancer screening: Advantages, controversies, and applications. Cancer Control 21: 9-14, 2014.

3. Bryant A and Cerfolio RJ: Differences in epidemiology, histology, and survival between cigarette smokers and never-smokers who develop non-small cell lung cancer. Chest 132: 185-192, 2007.

4. Nakamura $\mathrm{H}$ and Saji $\mathrm{H}$ : Worldwide trend of increasing primary adenocarcinoma of the lung. Surg Today 44: 1004-1012, 2014.

5. Kong J, Xu F, He M, Chen K and Qian B: The incidence of lung cancer by histological type: A population-based study in Tianjin, China during 1981-2005. Respirology 19: 1222-1228, 2014.

6. Cancer Genome Atlas Research Network: Comprehensive molecular profiling of lung adenocarcinoma. Nature 511: 543-550, 2014.

7. Devarakonda S, Morgensztern D and Govindan R: Genomic alterations in lung adenocarcinoma. Lancet Oncol 16: e342-e351, 2015.

8. Gautschi O, Hugli B, Ziegler A, Bigosch C, Bowers NL, Ratschiller D, Jermann M, Stahel RA, Heighway J and Betticher DC: Cyclin D1 (CCND1) A870G gene polymorphism modulates smoking-induced lung cancer risk and response to platinum-based chemotherapy in non-small cell lung cancer (NSCLC) patients. Lung Cancer 51: 303-311, 2006.

9. Li MY, Liu Y, Liu LZ, Kong AW, Zhao Z, Wu B, Long X, Wu J, Ng CS, Wan IY, et al: Estrogen receptor alpha promotes smoking-carcinogen-induced lung carcinogenesis via cytochrome P450 1B1. J Mol Med 93: 1221-1233, 2015.

10. Cheng Z, Wang W, Song YN, Kang Y and Xia J: hOGG1, p53 genes, and smoking interactions are associated with the development of lung cancer. Asian Pac J Cancer Prev 13: 1803-1808, 2012

11. Zhang J, Chen D, Shen QM, Tian DL, Jiang YH, Yin HN and Li HW: Association between cigarette smoking and FHIT gene alterations in Chinese lung cancer. Lung Cancer 29: 235, 2000.

12. Zhou W, Liu G, Park S, Wang Z, Wain JC, Lynch TJ, Su L and Christiani DC: Gene-smoking interaction associations for the ERCC1 polymorphisms in the risk of lung cancer. Cancer Epidemiol Biomarkers Prev 14: 491-496, 2005.

13. Zhou C, Chen H, Han L, Xue F, Wang A and Liang YJ: Screening of genes related to lung cancer caused by smoking with RNA-Seq. Eur Rev Med Pharmacol Sci 18: 117-125, 2014.

14. Kabbout M, Garcia MM, Fujimoto J, Liu DD, Woods D, Chow CW, Mendoza G, Momin AA, James BP, Solis L, et al: ETS2 mediated tumor suppressive function and MET oncogene inhibition in human non-small cell lung cancer. Clin Cancer Res 19: 3383-3395, 2013.

15. Landi MT, Dracheva T, Rotunno M, Figueroa JD, Liu H, Dasgupta A, Mann FE, Fukuoka J, Hames M, Bergen AW, et al: Gene expression signature of cigarette smoking and its role in lung adenocarcinoma development and survival. PLoS One 3: e1651, 2008. 
16. Ding L, Getz G, Wheeler DA, Mardis ER, McLellan MD, Cibulskis K, Sougnez C, Greulich H, Muzny DM, Morgan MB, et al: Somatic mutations affect key pathways in lung adenocarcinoma. Nature 455: 1069-1075, 2008.

17. Gautier L, Cope L, Bolstad BM and Irizarry RA: Affy-analysis of Affymetrix GeneChip data at the probe level. Bioinformatics 20: 307-315, 2004

18. Smyth GK: Limma: Linear models for microarray data. In: Bioinformatics and Computational Biology Solutions Using R and Bioconductor. Gentleman R, Carey V, Huber W, Irizarry R and Dudoit S (eds). Springer, New York, pp397-420, 2004.

19. Wang X, Kang DD, Shen K, Song C, Lu S, Chang LC, Liao SG, Huo Z, Tang S, Ding Y, et al: An R package suite for microarray meta-analysis in quality control, differentially expressed gene analysis and pathway enrichment detection. Bioinformatics 28: 2534-2536, 2012

20. Wang L, Cao C, Ma Q, Zeng Q, Wang H, Cheng Z, Zhu G, Qi J, $\mathrm{Ma} \mathrm{H}$, Nian $\mathrm{H}$ and Wang Y: RNA-seq analyses of multiple meristems of soybean: Novel and alternative transcripts, evolutionary and functional implications. BMC Plant Biol 14: 169, 2014

21. Keshava Prasad TS, Goel R, Kandasamy K, Keerthikumar S, Kumar S, Mathivanan S, Telikicherla D, Raju R, Shafreen B, Venugopal A, et al: Human protein reference database-2009 update. Nucleic Acids Res 37 (Database issue): D767-D772, 2009.

22. Smoot ME, Ono K, Ruscheinski J, Wang PL and Ideker T: Cytoscape 2.8: New features for data integration and network visualization. Bioinformatics 27: 431-432, 2011.

23. Barthélemy M: Betweenness centrality in large complex networks. Eur Phy J Conden Matter Com Sys 38: 163-168, 2004

24. Zhang HH, Ahn J, Lin X and Park C: Gene selection using support vector machines with non-convex penalty. Bioinformatics 22 88-95, 2006.

25. Brown MP, Grundy WN, Lin D, Cristianini N, Sugnet CW, Furey TS, Ares M Jr and Haussler D: Knowledge-based analysis of microarray gene expression data using support vector machines. Proc Natl Acad Sci USA 97: 262-267, 2000.

26. Stojanović M, Andjelković Apostolović M, Stojanović D, Milosević Z, Ignjatović A, Lakusić VM and Golubović M: Understanding sensitivity, specificity and predictive values Vojnosanit Pregl 71: 1062-1065, 2014.

27. Parikh R, Mathai A, Parikh S, Chandra Sekhar G and Thomas R: Understanding and using sensitivity, specificity and predictive values. Indian J Ophthalmol 56: 45-50, 2008.

28. Orru G, Pettersson-Yeo W, Marquand AF, Sartori G and Mechelli A: Using support vector machine to identify imaging biomarkers of neurological and psychiatric disease: A critica review. Neurosci Biobehav Rev 36: 1140-1152, 2012.

29. Fan XJ, Wan XB, Huang Y, Cai HM, Fu XH, Yang ZL, Chen DK, Song SX, Wu PH, Liu Q, et al: Epithelial-mesenchymal transition biomarkers and support vector machine guided model in preoperatively predicting regional lymph node metastasis for rectal cancer. Br J Cancer 106: 1735-1741, 2012.

30. Han M, Dai J, Zhang Y, Lin Q, Jiang M, Xu X, Liu Q and Jia J: Support vector machines coupled with proteomics approaches for detecting biomarkers predicting chemotherapy resistance in small cell lung cancer. Oncol Rep 28: 2233-2238, 2012.

31. Guyon I, Weston J and Barnhill S: Gene selection for cancer classification using support vector machines. Machine Learning 46: 389-422, 2002

32. Yousef M, Ketany M, Manevitz L, Showe LC and Showe MK Classification and biomarker identification using gene network modules and support vector machines. BMC Bioinformatics 10 : 337, 2009.

33. Guan P, Huang D, He M and Zhou B: Lung cancer gene expression database analysis incorporating prior knowledge with support vector machine-based classification method. J Exp Clin Cancer Res 28: 103, 2009.

34. Li P, Wang X, Liu Z, Liu H, Xu T, Wang H, Gomez DR, Nguyen QN, Wang LE, Teng Y, et al: Single nucleotide polymorphisms in CBLB, a regulator of T-cell response, predict radiation pneumonitis and outcomes after definitive radiotherapy for non-small-cell lung cancer. Clin Lung Cancer 17: 253-262, 2016.
35. Mu X, Zhang Y, Qu X, Hou K, Kang J, Hu X and Liu Y: Ubiquitin ligase Cbl-b is involved in icotinib (BPI-2009H)-induced apoptosis and G1 phase arrest of EGFR mutation-positive non-small-cell lung cancer. Biomed Res Int 2013: 726375, 2013.

36. Rountree MR, Bachman KE, Herman JG and Baylin SB: DNA methylation, chromatin inheritance, and cancer. Oncogene 20: 3156-3165, 2001

37. Kerr KM, Galler JS, Hagen JA, Laird PW and Laird-Offringa IA The role of DNA methylation in the development and progression of lung adenocarcinoma. Dis Markers 23: 5-30, 2007.

38. Lin RK, Hsieh YS, Lin P, Hsu HS, Chen CY, Tang YA, Lee CF and Wang YC: The tobacco-specific carcinogen NNK induces DNA methyltransferase 1 accumulation and tumor suppressor gene hypermethylation in mice and lung cancer patients. J Clin Invest 120: 521-532, 2010.

39. Tennis MA, Vanscoyk MM, Wilson LA, Kelley N and Winn RA: Methylation of Wnt7a is modulated by DNMT1 and cigarette smoke condensate in non-small cell lung cancer. PLoS One 7: e32921, 2012.

40. McCabe MT and Creasy CL: EZH2 as a potential target in cancer therapy. Epigenomics 6: 341-351, 2014.

41. Yoon KA, Gil HJ, Han J, Park J and Lee JS: Genetic polymorphisms in the polycomb group gene EZH2 and the risk of lung cancer. J Thorac Oncol 5: 10-16, 2010.

42. Zhang H, Zhang H, Zhao M, Lv Z, Zhang X, Qin X, Wang H, Wang S, Su J, Lv X, et al: miR-138 inhibits tumor growth through repression of EZH2 in non-small cell lung cancer. Cell Physiol Biochem 31: 56-65, 2013

43. Xia H, Zhang W, Li Y, Guo N and Yu C: EZH2 silencing with RNA interference induces G2/M arrest in human lung cancer cells in vitro. Biomed Res Int 2014: 348728, 2014.

44. Wang X, Zhao H, Lv L, Bao L, Wang X and Han S: Prognostic significance of EZH2 expression in non-small cell lung cancer: A meta-analysis. Sci Rep 6: 19239, 2016.

45. Zhang C, Ge S, Hu C, Yang N and Zhang J: miRNA-218, a new regulator of HMGB1, suppresses cell migration and invasion in non-small cell lung cancer. Acta Biochim Biophys Sin (Shanghai) 45: 1055-1061, 2013.

46. Shen X, Hong L, Sun H, Shi M and Song Y: The expression of high-mobility group protein box 1 correlates with the progression of non-small cell lung cancer. Oncol Rep 22: 535-539, 2009.

47. Zheng W, Jiang C and Li R: Integrin and gene network analysis reveals that ITGA5 and ITGB1 are prognostic in non-small-cell lung cancer. Onco Targets Ther 9: 2317-2327, 2016.

48. Jono $\mathrm{H}$ and Ando Y: Midkine: A novel prognostic biomarker for cancer. Cancers 2: 624-641, 2010.

49. Yuan K, Chen Z, Li W, Gao CE, Li G, Guo G, Yang Y, Ai Y, $\mathrm{Wu} \mathrm{L}$ and Zhang M: MDK protein overexpression correlates with the malignant status and prognosis of non-small cell lung cancer. Arch Med Res 46: 635-641, 2015.

50. Hao H, Maeda Y, Fukazawa T, Yamatsuji T, Takaoka M, Bao XH, Matsuoka J, Okui T, Shimo T, Takigawa N, et al: Inhibition of the growth factor MDK/midkine by a novel small molecule compound to treat non-small cell lung cancer. PLoS One 8: e71093, 2013.

51. Regala RP, Davis RK, Kunz A, Khoor A, Leitges M and Fields AP: Atypical protein kinase $\mathrm{C}\{$ iota $\}$ is required for bronchioalveolar stem cell expansion and lung tumorigenesis. Cancer Res 69: 7603-7611, 2009.

52. Sutterlüty H, Mayer CE, Attems J, Setinek U, Mikula M, Mikulits W, Micksche M and Berger W: Inhibition of cell migration and proliferation in non-small cell lung cancer (NSCLC) by Sprouty 2 (Spry2) via K-Ras dependent and independent pathways. Cancer Res 66 (Suppl 8): S349-S350, 2006.

53. Sutterlüty H, Mayer CE, Setinek U, Attems J, Ovtcharov S, Mikula M, Mikulits W, Micksche $M$ and Berger W: Down-regulation of Sprouty2 in non-small cell lung cancer contributes to tumor malignancy via extracellular signal-regulated kinase pathway-dependent and -independent mechanisms. Mol Cancer Res 5: 509-520, 2007.

This work is licensed under a Creative Commons Attribution-NonCommercial-NoDerivatives 4.0 International (CC BY-NC-ND 4.0) License. 